\title{
Quasiperiodic, periodic, and slowing-down states of coupled heteroclinic cycles
}

\author{
Dong Li, ${ }^{1}$ M. C. Cross, ${ }^{2}$ Changsong Zhou, ${ }^{3}$ and Zhigang Zheng ${ }^{1, *}$ \\ ${ }^{1}$ Department of Physics and the Beijing-Hong Kong-Singapore Joint Center for Nonlinear and Complex Systems (Beijing), \\ Beijing Normal University, Beijing 100875, China \\ ${ }^{2}$ Department of Physics, California Institute of Technology, Pasadena, California 91125, USA \\ ${ }^{3}$ Department of Physics, Centre for Nonlinear Studies and Beijing-Hong Kong-Singapore Joint Centre for Nonlinear and Complex Systems \\ (Hong Kong), Hong Kong Baptist University, Kowloon Tong, Hong Kong, China
}

(Received 6 July 2011; revised manuscript received 28 November 2011; published 30 January 2012)

\begin{abstract}
We investigate two coupled oscillators, each of which shows an attracting heteroclinic cycle in the absence of coupling. The two heteroclinic cycles are nonidentical. Weak coupling can lead to the elimination of the slowing-down state that asymptotically approaches the heteroclinic cycle for a single cycle, giving rise to either quasiperiodic motion with separate frequencies from the two cycles or periodic motion in which the two cycles are synchronized. The synchronization transition, which occurs via a Hopf bifurcation, is not induced by the commensurability of the two cycle frequencies but rather by the disappearance of the weaker frequency oscillation. For even larger coupling the motion changes via a resonant heteroclinic bifurcation to a slowing-down state corresponding to a single attracting heteroclinic orbit. Coexistence of multiple attractors can be found for some parameter regions. These results are of interest in ecological, sociological, neuronal, and other dynamical systems, which have the structure of coupled heteroclinic cycles.
\end{abstract}

DOI: 10.1103/PhysRevE.85.016215

PACS number(s): 05.45.Xt

\section{INTRODUCTION}

A heteroclinic cycle is an invariant set consisting of the union of a set of saddles $\left\{S_{1}, \ldots, S_{n}\right\}$ and the trajectories backward asymptotic to each saddle $S_{i}$ and forward asymptotic to the next one $S_{i+1}$ [1]. Such a structure has been frequently found in neuronal dynamics [2-7], fluid mechanics [8-10], ecology [11-14], sociology [15-19], and other dynamical systems. A heteroclinic cycle has some significantly different characteristics from other types of dynamical states, such as limit cycles or chaotic attractors. One of these characteristics is the slowing-down effect: When the trajectory gets close to the heteroclinic cycle, the system may spend a longer and longer time in the vicinity of each saddle before quickly switching to the vicinity of the next saddle. The residence time in the vicinity of each saddle increases exponentially, and the oscillation slows down as the return time diverges $[10,20]$. The slowing-down effect can be avoided by introducing external noise [2,21-23] or diffusion terms [10] to the heteroclinic cycle. When systems displaying heteroclinic cycles are coupled, they can show rich nontrivial behaviors [1,24-27], which are significant in demonstrating and understanding a wide range of interesting phenomena in different fields. Synchronization, one of the most characteristic collective behaviors in dynamical systems [28-37], has also been reported in a periodically driven heteroclinic cycle system [2] and in experiments on two coupled subsystems with heteroclinic cycle structures [5].

Recently the dynamical behavior of a small number of coupled systems that have the structure of heteroclinic cycles has attracted increasing interest [5-7,38-40]. For example, synchronization of two coupled heteroclinic cycles is of significance for understanding the microcircuit dynamics

*zgzheng@bnu.edu.cn in neuronal systems [5]. The inhibitory neurons and the asymmetry of the inhibitory connections can make the typical dynamics of a microcircuit process a heteroclinic cycle [2]. Synchronization makes it possible for the microcircuits to coordinate with each other [5]. In another example, in studies of the mechanism of the coexistence of a large number of competing species in a basically homogeneous environment, it is valuable to consider the case that the habitat is subdivided into two patches [38-40]. The dynamics of two coupled heteroclinic cycles can therefore contribute to the exploration of the fundamental mechanism underlying the phenomena of coexisting species and more complex situations.

In this paper we study in detail the dynamical behavior of two coupled subsystems, each of which shows an attracting heteroclinic cycle in the absence of coupling. These two heteroclinic cycles are nonidentical. We show that interactions between these two cycles can change their dynamical behavior to avoid the slowing-down state and the sensitivity to small perturbations. In the case of weak interactions, the oscillatory dynamics can be maintained just as in the case of added noise, but now with two independent periodic cycles of incommensurate frequencies. When the interactions become stronger, a range of interesting behavior is found, including a slowing-down state, coexistence of multiple attractors, and synchronization. Interestingly, the synchronization transition is not induced by the commensurability of the two cycle frequencies but rather by the disappearance of the weaker frequency oscillation, and the synchronized frequency can be either bigger or smaller than both the desynchronized frequencies.

\section{HETEROCLINIC CYCLE MODEL}

We start our study from the model proposed by Busse and Heikes [8] to describe the turbulence in a rotating layer 
of convecting fluid, which consists of three variables $A_{i}(i=$ 1, 2, 3):

$$
\left\{\begin{array}{l}
\tau d A_{1} / d t=A_{1}\left(\varepsilon-A_{1}^{2}-g_{+} A_{2}^{2}-g_{-} A_{3}^{2}\right), \\
\tau d A_{2} / d t=A_{2}\left(\varepsilon-A_{2}^{2}-g_{+} A_{3}^{2}-g_{-} A_{1}^{2}\right), \\
\tau d A_{3} / d t=A_{3}\left(\varepsilon-A_{3}^{2}-g_{+} A_{1}^{2}-g_{-} A_{2}^{2}\right) .
\end{array}\right.
$$

The dynamics of the model is a typical heteroclinic cycle passing through the saddles at $A_{i}=\sqrt{\varepsilon}$ for positive initial conditions. The typical slowing-down effect can be found in this model: The residence time in the vicinity of each saddle increases exponentially as time progresses, and the oscillation slows down.

Equation (1) has the typical form of a broad range of heteroclinic cycle systems, so that its dynamical behavior is of general significance. For example, it has the same form as a Gause-Lotka-Volterra-type three-competitors system [20], where the variables can represent the population densities in ecology systems, or the rates of neuronal activity in neuronal systems [2].

In this paper we discuss two interacting heteroclinic cycles $\left(A_{1}, A_{2}, A_{3}\right)$ and $\left(B_{1}, B_{2}, B_{3}\right)$. We do not wish to induce too much asymmetry. Thus, the choice of coupling should preserve the symmetry of simultaneously alternating the coordinates of the two subsystems in turn $\left(A_{1}, B_{1} \rightarrow A_{2}, B_{2} \rightarrow A_{3}, B_{3} \rightarrow\right.$ $\left.A_{1}, B_{1}\right)$. A simple choice is linear coupling, in which case the equations of motion can be written as follows:

$$
\left\{\begin{array}{l}
\tau_{a} d A_{1} / d t=A_{1}\left(\varepsilon_{a}-A_{1}^{2}-g_{+} A_{2}^{2}-g_{-} A_{3}^{2}\right)+\xi\left(B_{1}-A_{1}\right), \\
\tau_{a} d A_{2} / d t=A_{2}\left(\varepsilon_{a}-A_{2}^{2}-g_{+} A_{3}^{2}-g_{-} A_{1}^{2}\right)+\xi\left(B_{2}-A_{2}\right), \\
\tau_{a} d A_{3} / d t=A_{3}\left(\varepsilon_{a}-A_{3}^{2}-g_{+} A_{1}^{2}-g_{-} A_{2}^{2}\right)+\xi\left(B_{3}-A_{3}\right), \\
\tau_{b} d B_{1} / d t=B_{1}\left(\varepsilon_{b}-B_{1}^{2}-g_{+} B_{2}^{2}-g_{-} B_{3}^{2}\right)+\xi\left(A_{1}-B_{1}\right), \\
\tau_{b} d B_{2} / d t=B_{2}\left(\varepsilon_{b}-B_{2}^{2}-g_{+} B_{3}^{2}-g_{-} B_{1}^{2}\right)+\xi\left(A_{2}-B_{2}\right), \\
\tau_{b} d B_{3} / d t=B_{3}\left(\varepsilon_{b}-B_{3}^{2}-g_{+} B_{1}^{2}-g_{-} B_{2}^{2}\right)+\xi\left(A_{3}-B_{3}\right),
\end{array}\right.
$$

We are interested in the effect of coupling on the dynamical behavior of heteroclinic cycles. In the following discussion, we study the coupling-induced oscillation phenomena in Sec. III, and the underlying mechanism in Sec. IV.

\section{QUASIPERIODIC AND PERIODIC OSCILLATIONS}

We first investigate the model Eq. (2) by fixing parameters $\tau_{a}=\tau_{b}=1, \varepsilon_{a}=1$, and $\varepsilon_{b}=4$ and vary the coupling strength $\xi$. These values of $\tau_{a, b}$ and $\varepsilon_{a, b}$ guarantee a reasonable difference between these two cycles.

In the absence of coupling, $\xi=0$, the two cycles $\left(A_{1}, A_{2}, A_{3}\right)$ and $\left(B_{1}, B_{2}, B_{3}\right)$ exhibit the slowing-down behavior. On the other hand, for $\xi \neq 0$, even if $\xi$ is very small, we find the interesting phenomenon that the slowing-down dynamics is eliminated; instead the dynamics display a two-frequency quasiperiodic motion. When the coupling is increased beyond a first critical value $\xi_{c}$, the system arrives at a periodic oscillatory state-a type of synchronized state. When the coupling strength is further increased beyond a second critical value $\xi_{c}^{\prime}$, the system changes to another slowing-down state corresponding to an attracting single heteroclinic cycle in six-dimensional space. This can be viewed as the second type of synchronized state in the system.
In the following, we show the bifurcation process and related nonlinear dynamics in detail. The range of dynamical behavior of the system (2) for small coupling $\xi$ is shown in Fig. 1. In the first row of Fig. 1 the coupling is $\xi=5 \times 10^{-5}$. Panel (a) shows the evolution of $A_{1}(t)$. In order to give a more direct impression of the dynamical behavior, in panel (b) we project the trajectory of the variables $A_{i}$ of the first cycle onto the two-dimensional $X_{1}-Y_{1}$ plane defined by

$$
\left\{\begin{array}{l}
X_{1}=\left|A_{1}\right|+\left|A_{2}\right| \cos 2 \pi / 3+\left|A_{3}\right| \cos 4 \pi / 3, \\
Y_{1}=\left|A_{2}\right| \sin 2 \pi / 3+\left|A_{3}\right| \sin 4 \pi / 3 ;
\end{array}\right.
$$

i.e., we let the projections of the three variables $A_{i}$ uniformly distribute around the origin with relative orientation $2 \pi / 3$ to each other. The behavior of each oscillator is similar to that of a single heteroclinic cycle driven by small noise, in that the trajectory stays near each saddle for a long time. In contrast to a periodic state, the residence times near the saddles vary from cycle to cycle. The inset of panel (b) expands the region near the saddle showing that the trajectory is not a limit cycle but is quasiperiodic motion. To show this more clearly, a Poincaré section satisfying $A_{1}=A_{2}, A_{3}>A_{2}$ is constructed, as in panel (c). A loop on this section demonstrates the two-frequency quasiperiodic nature of the oscillation. The Fourier transforms of the time series of $A_{1}$ and $B_{1}$ are shown in panel (d). The two oscillators show the same peaks, albeit with different intensities.

The full range of behavior as the coupling strength is varied is shown in Fig. 2 by plotting the dependence of the periods $2 \pi / \omega_{i}$ against $\log _{10} \xi$. Like the average period induced by noise [21], for small values of $\xi$ the dependence approximates to straight lines until a critical point $\xi_{c}$, where we find that the weaker oscillation disappears.

To compare the case of small coupling with that influenced by noise, we show some results of simulations in the presence of noise rather than the coupling terms. A Gaussian white-noise term $\eta_{i}(t)$ is added to each equation for $\tau d A_{i} / d t$ of Eq. (1). To relate to the system (2) where the coupling terms in the three dimensions are not independent, we correlate $\eta_{i}(t)$ in the form

$$
\left\{\begin{array}{l}
\eta_{1}(t)=\zeta(t) \cos \theta \\
\eta_{2}(t)=\zeta(t) \cos (\theta-2 \pi / 3) \\
\eta_{3}(t)=\zeta(t) \cos (\theta-4 \pi / 3)
\end{array}\right.
$$

where $\xi(t)$ is taken from a Gaussian distribution

$$
\left\{\begin{array}{l}
\langle\zeta(t)\rangle=0 \\
\langle\zeta(t) \zeta(s)\rangle=2 D \delta(t-s)
\end{array}\right.
$$

and $\theta$ is taken from a uniform distribution $f(\theta)$ from 0 to $\pi$ :

$$
f(\theta)=\left\{\begin{array}{ll}
\frac{1}{\pi}, & 0<\theta \leqslant \pi \\
0, & \text { otherwise }
\end{array} .\right.
$$

In this case, the dynamics of the single cycle can be driven away from the slowing-down state to maintain the oscillatory motion. Stone and Holmes [21] proved that the average period $\langle T\rangle$ is a linear function of the logarithmic noise intensity:

$$
\langle T\rangle=K_{0}+K_{1}\left|\log _{10} D\right| .
$$



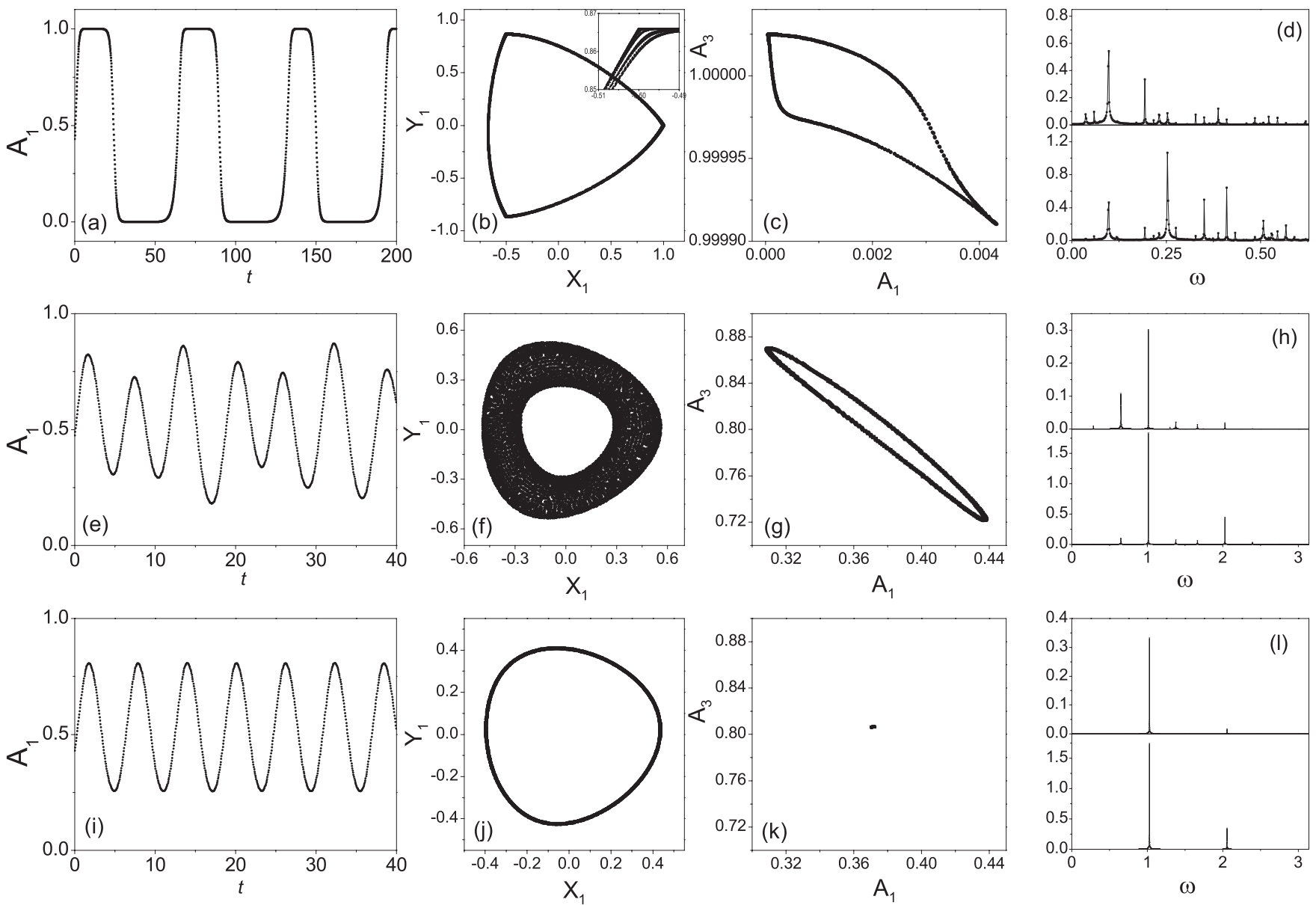

FIG. 1. Dynamics of two coupled heterocycles [Eq. (2)] for different coupling strengths: $\xi=5 \times 10^{-5}$ for the first row (a)-(d); $\xi=0.09072$, which is slightly smaller than the critical value for the bifurcation to a periodic orbit, for the second row (e)-(h); and $\xi=0.09537$, which is slightly larger than the critical value, for the third (i)-(1). The first column shows the time series. The second column shows the projections of the phase space onto the $X_{1}-Y_{1}$ plane, with the inset in panel (b) expanding the region near a saddle to show the quasiperiodic nature of the orbit. The third column exhibits the Poincare section: $A_{1}=A_{2}, A_{3}>A_{2}$. The fourth column shows the Fourier transform of the two oscillators. Other parameter values are $\tau_{a}=\tau_{b}=1, \varepsilon_{a}=1, \varepsilon_{b}=4, g_{-}=0.5$, and $g_{+}=2$.

This is illustrated by numerically simulating Eqs. (1) and (4)(6) in Fig. 3. We define a phase variable $\phi(t)$ of the trajectory as

$$
\phi=\arctan \frac{Y}{X}+n \pi,
$$

and then the average period is $\lim _{t \rightarrow \infty} 2 \pi t / \phi$.

In the case of a small coupling between two cycles, the coupling terms play a vital role in modifying the principal frequency of the oscillator in a similar way to the mean effect of small noise. By comparing Figs. 2 and 3, one can notice that the linear dependence of the period on the logarithmic coupling strength is very similar to the dependence on the logarithmic noise strength for a single noisy cycle. However, from another point of view, the coupling is also sufficiently weak that the two cycles have independent frequencies.

As the coupling strength $\xi$ increases, the dynamics of the coupled cycles becomes distinctly different from that of the single heteroclinic cycle, and the saddle no longer plays a prominent role, as shown in the second row of Fig. 1 for $\xi=$ 0.09072 , panels $(\mathrm{e})-(\mathrm{h})$. The motion is again quasiperiodic, but now the same frequency dominates the power spectrum for both cycles-for the parameters used, with $\varepsilon_{b}=4$ larger than $\varepsilon_{a}=1$, the frequency of the stronger power belongs to the second cycle.

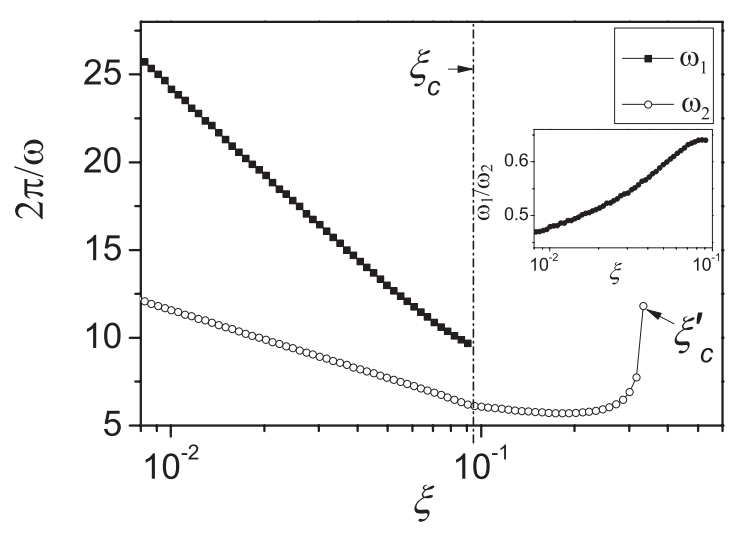

FIG. 2. Dependence of the periods $2 \pi / \omega$ on the logarithm of the coupling strength $\log _{10} \xi$. Parameter values for the single cycle are the same as in Fig. 1. The inset shows the ratio of the two frequencies in the quasiperiodic state. 


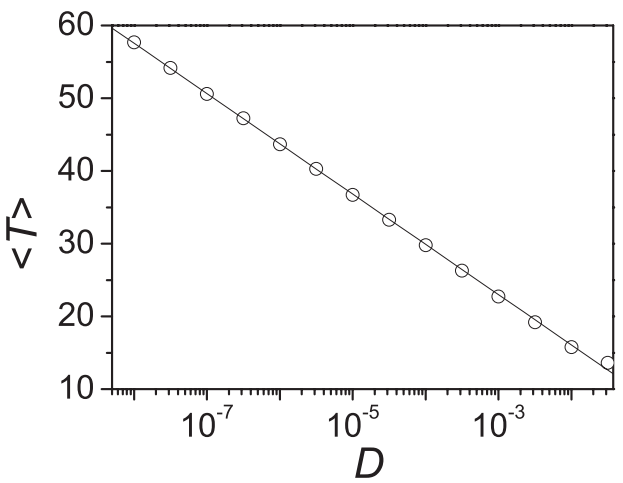

FIG. 3. Average period of a single heteroclinic cycle with added noise, Eqs. (1) and (4)-(6). The period exhibits a linear dependence on the logarithmic noise intensity. The parameters are $\varepsilon=1$, with other values the same as in Fig. 2. Fitted values for $K_{0}$ and $K_{1}$ of Eq. (7) are 2.242 and 6.916, respectively.

When the coupling strength is increased to some critical value $\xi_{c}$, synchronization is achieved so that the dynamics of the two cycles become periodic, as shown in the third row of Fig. 1, panels (i)-(1). This bifurcation does not come from the commensurability of the two frequencies but from the disappearance of the weaker oscillation frequency. The development of a second frequency with a continuously growing amplitude (as the coupling is decreased) is a typical Hopf bifurcation from a periodic orbit.

In order to give a more direct impression of the synchronization phenomenon, we show in Fig. 4 the correlation between the $X$ variables of the two cycles for the same three values of $\xi$ as in Fig. 1. When $\xi$ is small, the coupling terms play a role similar to noise, so that the two cycles exhibit a weak correlation. Just below the critical coupling $\xi_{c}$ for the bifurcation to the periodic state, the frequency from the stronger subsystem dominates both cycles, so that the quasiperiodic behavior is also obvious on the $X_{2}-X_{1}$ plane. The periodic oscillation in the synchronized state, where $X_{2}$ exhibits a simple curve against $X_{1}$, is typical phase synchronization, where a Hopf bifurcation occurs at the transition [33]. The difference here is that the stronger subsystem dominates, while the two oscillators usually play equal roles in the other cases of phase synchronization of linearly coupled limit-cycle oscillators [41] (e.g., the synchronized frequency in these examples is the mean of the separate frequencies) or chaotic oscillators [33]. This type of synchronization behavior has some similarities to generalized synchronization [32], where the dynamical variables from one system turn out to be a function of the variables of another one. The present case is similar in that the slave (weaker) cycle strictly follows the master (stronger) cycle.

The synchronization behavior in our work is also obviously different from the case where the cycle is forced by a periodic input as analyzed by Rabinovich et al. [2]. In the present case, there is no intrinsic frequency when the coupling $\xi=0$ : The emergent frequencies originate from the coupling effect.

In Fig. 2, when $\xi<\xi_{c}$, the ratio of the two angular frequencies monotonically increases, as shown in the inset. When the coupling strength $\xi$ is increased above the critical value $\xi_{c}$, the two coupled cycles possess the same period in the
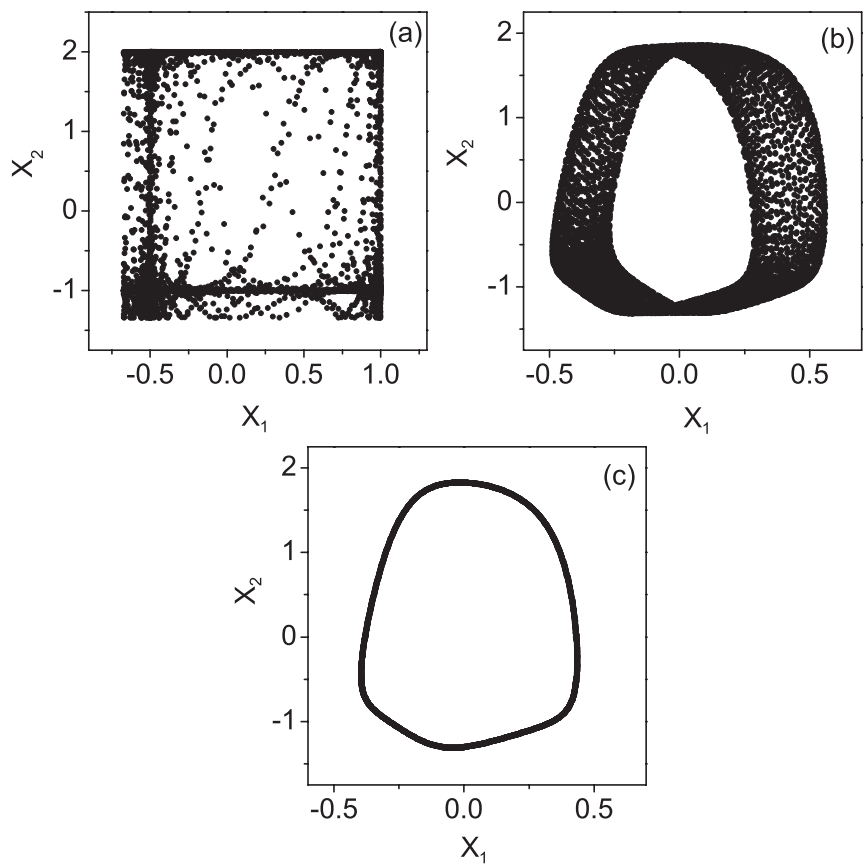

FIG. 4. Dynamics on the $X_{2}-X_{1}$ plane for the same three values of $\xi$ and the same values of the other parameters as in Fig. 1.

synchronized state. The synchronized frequency can be larger than both of the desynchronized frequencies. However, when the coupling becomes stronger, the synchronized frequency decreases to a small value, as shown in Fig. 2 for $\xi>0.3$. We will reveal the mechanism underlying the change of the synchronized frequency in the next section.

Szücs et al. [5] have reported that when two neuronal microcircuit oscillators are coupled, the synchronized frequency can be either larger or smaller than both of the desynchronized frequencies, depending on the form of the coupling. In our work, we use a coupling form different from any one used in Ref. [5] but also observe the upward and downward shifts of synchronized frequency. We will show in the next section that the upward (or downward) shift of synchronized frequency is induced by the increasing (or decreasing) distance of the trajectory to the saddles in phase space. We can therefore understand the results in Ref. [5] as depending on whether this coupling form used tends to increase (or decrease) the distance of the trajectory to the saddles in phase space.

\section{SLOWING-DOWN STATE AND COEXISTENCE OF MULTIPLE ATTRACTORS}

As the coupling $\xi$ increases further toward another critical value $\xi_{c}^{\prime}$, the coupled cycles again approach a slowing-down state, implying that a heteroclinic cycle in the six-dimensional space becomes stable. In Fig. 2 the period slows down at the second critical value $\xi_{c}^{\prime} \approx 0.3394$. A periodic orbit for $\xi=0.33$ just below this critical value and a slowing-down state for $\xi=0.35$ above the critical value are shown in Fig. 5 .

In order to identify the heteroclinic cycle in the sixdimensional space, we begin the analysis with the fixed point structure of the coupled dynamical equations (2). First of all, the fixed points $S_{i}\left(i=1,2,3, A_{j} \equiv \tilde{A_{j}} \neq 0\right.$, and $B_{j} \equiv$ $\tilde{B}_{j} \neq 0$ for $j=i, A_{j}=B_{j}=0$ for $j \neq i$ ) are determined. 
We describe the behavior near the $S_{1}$ fixed point; the behavior near the other fixed points follows by cyclically permuting the indices, since the structure of the three fixed points is identical. By introducing the ratio of amplitudes $c_{1}$ at the fixed point $S_{1}$ so that $\tilde{B}_{1}=c \tilde{A_{1}}$, one gets

$$
\left\{\begin{array}{l}
\varepsilon_{a}+\xi\left(c_{1}-1\right)={\tilde{A_{1}}}^{2}, \\
\varepsilon_{b}+\xi\left(1-c_{1}\right) / c_{1}=\tilde{B}_{1}{ }^{2}, \\
c_{1}^{2}{\tilde{A_{1}}}^{2}=\tilde{B}_{1}{ }^{2},
\end{array}\right.
$$

and the three quantities $c_{1}, \tilde{A}_{1}$, and $\tilde{B_{1}}$ can be obtained by solving these equations.

Choosing $\tau_{a}=\tau_{b}=1$ as before, the linear stability equation near this fixed point is

$$
\frac{d}{d t}\left(\begin{array}{l}
\delta A_{1} \\
\delta B_{1} \\
\delta A_{2} \\
\delta B_{2} \\
\delta A_{3} \\
\delta B_{3}
\end{array}\right)=\left(\begin{array}{lll}
M_{1} & & \\
& M_{2} & \\
& & M_{3}
\end{array}\right)\left(\begin{array}{l}
\delta A_{1} \\
\delta B_{1} \\
\delta A_{2} \\
\delta B_{2} \\
\\
\end{array}\right.
$$

where the submatrices are

$$
M_{1}=\left(\begin{array}{cc}
\varepsilon_{a}-3{\tilde{A_{1}}}^{2}-\xi & \xi \\
\xi & \varepsilon_{b}-3 \tilde{B}_{1}^{2}-\xi
\end{array}\right)
$$

with two eigenvalues $\lambda_{11}>\lambda_{12}$,

$$
M_{2}=\left(\begin{array}{cc}
\varepsilon_{a}-g_{-}{\tilde{A_{1}}}^{2}-\xi & \xi \\
\xi & \varepsilon_{b}-g_{-}{\tilde{B_{1}}}^{2}-\xi
\end{array}\right)
$$

with two eigenvalues $\lambda_{21}>\lambda_{22}$, and

$$
M_{3}=\left(\begin{array}{cc}
\varepsilon_{a}-g_{+}{\tilde{A_{1}}}^{2}-\xi & \xi \\
\xi & \varepsilon_{b}-g_{+} \tilde{B}_{1}^{2}-\xi
\end{array}\right)
$$

with two eigenvalues $\lambda_{31}>\lambda_{32}$. In each two-dimensional subspace $\left\{\delta A_{i}, \delta B_{i}\right\}$, the length of a vector grows at a rate determined by the larger eigenvalue, i.e., proportional to $\exp \left(\lambda_{i 1} t\right)$, and the ratio of $B_{i}$ to $A_{i}$ for the components growing away from the fixed point is close to the value given by the corresponding eigenvector, as shown in Fig. 5.

In the parameter region of the slowing-down state of system (1), both $\lambda_{11}$ and $\lambda_{12}$ are negative, corresponding to an attracting four-dimensional surface in which the two cycles are embedded. Moreover, $\lambda_{21}>0$, and $\lambda_{31}<0$, corresponding to a divergence of the vector in the subspace $\left\{\delta A_{2}, \delta B_{2}\right\}$ and a convergence of the vector in the subspace $\left\{\delta A_{3}, \delta B_{3}\right\}$. Thus this fixed point is a saddle. There are corresponding results for $S_{2}$ and $S_{3}$, and heteroclinic orbits directly linking the three saddles again form a heteroclinic cycle.

The eigenvalues $\lambda_{22}$ and $\lambda_{32}$ also play important roles in the attractors in such type of dynamical systems [42]. In our system, $\lambda_{32}$ is always negative, but $\lambda_{22}$ can be either negative or positive, depending on the coupling strength. We first discuss the case $\lambda_{22}<0$.

When $\lambda_{22}<0$, the dynamical behavior near the saddles is almost the same as that near a saddle of the system (1). This heteroclinic cycle in the six-dimensional space can be proven to be structurally stable in this case, since each of the

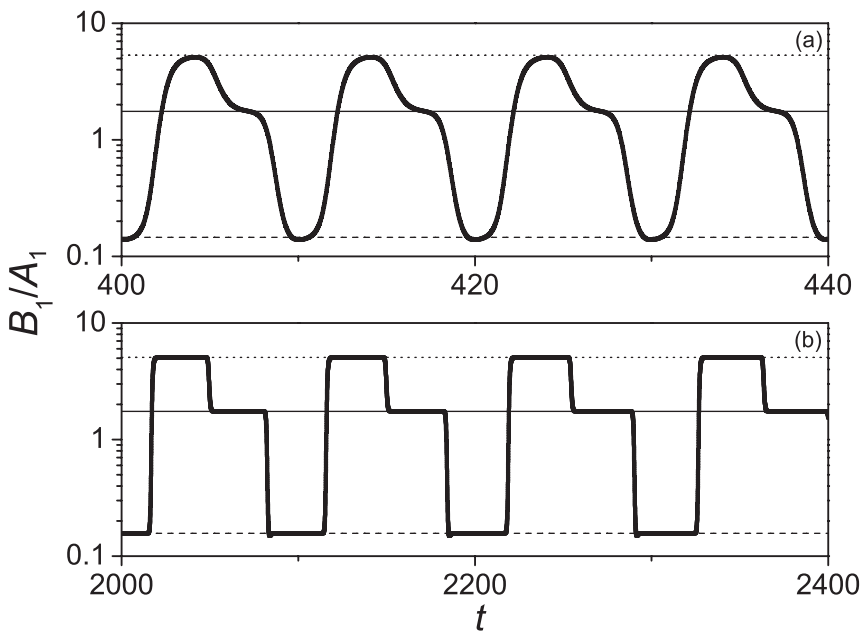

FIG. 5. Ratio of $B_{1}$ to $A_{1}$ for the periodic orbit at (a) $\xi=0.33$ and (b) $\xi=0.35$. Other parameters are the same as Fig. 3. The horizontal lines are theoretical predictions of the ratio near the saddles. Noticing that there are two constant values of $B_{1} / A_{1}$ given by the eigenvectors in each subspace $\left\{A_{i}, B_{i}\right\}$ near the saddle $S_{i}$, the dashed and dotted lines are, respectively, the values given by the eigenvectors corresponding to the larger eigenvalue near $S_{2}$ and $S_{3}$. The solid line is the value $c_{1}$ calculated from Eq. (9) near the saddle $S_{1}$.

heteroclinic orbits lies within a symmetry-invariant subspace $\left(A_{i}, B_{i}, A_{i+1}, B_{i+1}\right.$ ) (where $A_{i+1}$ and $B_{i+1}$ are defined as $A_{1}$ and $B_{1}$ when $\left.i=3\right)$. In the subspace $\left(A_{1}, B_{1}, A_{2}, B_{2}\right)$ for example, the heteroclinic orbit directionally connects $S_{1}$ to $S_{2} . S_{1}$ is a saddle in this four-dimensional subspace. It has a three-dimensional stable manifold corresponding to the negative eigenvalues $\lambda_{11}, \lambda_{12}$, and $\lambda_{22}$, and a one-dimensional unstable manifold corresponding to the positive eigenvalue $\lambda_{21} . S_{2}$ is a sink in this subspace, since the eigenvalues $\lambda_{31}, \lambda_{32}$, $\lambda_{11}$, and $\lambda_{12}$ are all negative. According to the method of Krupa [43], such a heteroclinic orbit lying within symmetry-invariant subspaces is structurally stable.

The structurally stable heteroclinic cycle of the last paragraph can be repelling or attracting, depending on the parameter values. We can find the condition for the cycle to be attracting following the method of Krupa and Melbourne [25], although the analysis is simpler in the present case because our saddles are identical. The condition for the cycle to be attracting is

$$
\lambda_{21}+\lambda_{31}<0 \text {. }
$$

The role of the heteroclinic cycle on varying the coupling $\xi$ is displayed in Fig. 6 by giving the value of $\tilde{B}_{1}$ at the fixed point $S_{1}$ and the range of $B_{1}$ in the trajectory near $S_{1}$ for the same parameter values used in Fig. 2. As we have seen, when the coupling increases, the system bifurcates from quasiperiodic to periodic motion and then back to the slowing-down state. During these transitions, the size of the heteroclinic cycle (measured by the value of $\tilde{B}_{1}$ ) becomes monotonically smaller, as shown in Fig. 6. Meanwhile, initially the amplitude of the quasiperiodic orbit shrinks more rapidly, so that the trajectory moves away from the unstable heteroclinic cycle. After the quasiperiodic orbit bifurcates to the periodic orbit, 


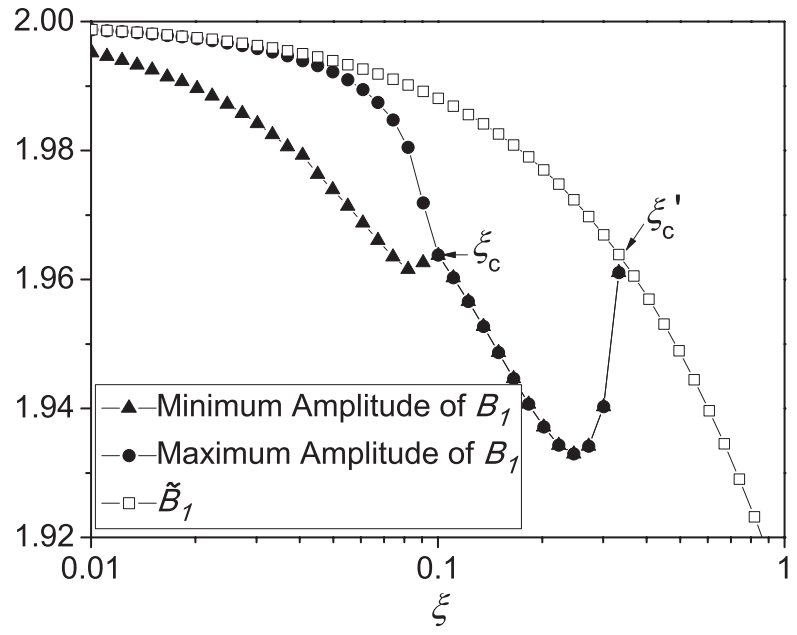

FIG. 6. Values of the amplitude $\tilde{B}_{1}$ at the saddle $S_{1}$ and the minimum and maximum amplitudes of $B_{1}$ in the trajectory near the saddle $S_{1}$ as a function of the coupling strength $\xi$. The values $\xi_{c}$ and $\xi_{c}^{\prime}$ give the bifurcation from quasiperiodic to periodic, and from periodic to the slowing-down state, respectively.

its amplitude eventually begins to increase, until it touches the heteroclinic cycle. This is the resonant heteroclinic bifurcation point given by Eq. (14) at which the cycle becomes attracting, and for larger couplings $\xi$ the heteroclinic cycle becomes the only attractor. Comparing Fig. 6 and Fig. 2, one can easily understand that the upward (or downward) shift of synchronized frequency is induced by the increasing (or decreasing) value of the distance between the trajectory and the saddles in phase space (the distance between filled points and unfilled points in Fig. 6), consistent with the analysis by Stone and Holmes [21].

Considering this bifurcation process in the reverse direction (decreasing $\xi$ ), the sequence of dynamical behaviors looks similar to the case where periodic or quasiperiodic oscillations are induced by the intermittent dynamics from a heteroclinic cycle $[44,45]$. In those systems, the heteroclinic cycle can be derived by ignoring the small higher-order terms. When these terms are taken into consideration, intermittent dynamics are induced, including chaos, quasiperiodic, and periodic solutions [44]. However, in our system, the violation of Eq. (14) gives a resonant heteroclinic bifurcation, so that the heteroclinic cycle just loses its stability, in contrast to the more familiar case where the heteroclinic cycle is destroyed by higher-order terms or other kinds of perturbations. Furthermore, in our work, we focus on the interaction between the two subsystems, rather than the perturbations that emerge when reducing a general system to a heteroclinic cycle structure, so as to be more relevant to the collective behavior of two coupled systems, in each of which there is an attracting heteroclinic cycle.

When $\lambda_{22}>0$, the situation becomes more complicated. In this domain we numerically find that even when the heteroclinic cycle is stable, stable quasiperiodic or periodic orbits may also exist, so that there can be multiple attractors. The phase diagram on varying both $\xi$ and $\varepsilon_{b}$ is displayed in Fig. 7. (Figures 2 and 6 correspond to a cut at $\varepsilon_{b}^{1 / 2}=$ 2.) For large $\varepsilon_{b}$, increasing the coupling gives transitions from quasiperiodic to periodic oscillations, and finally to the

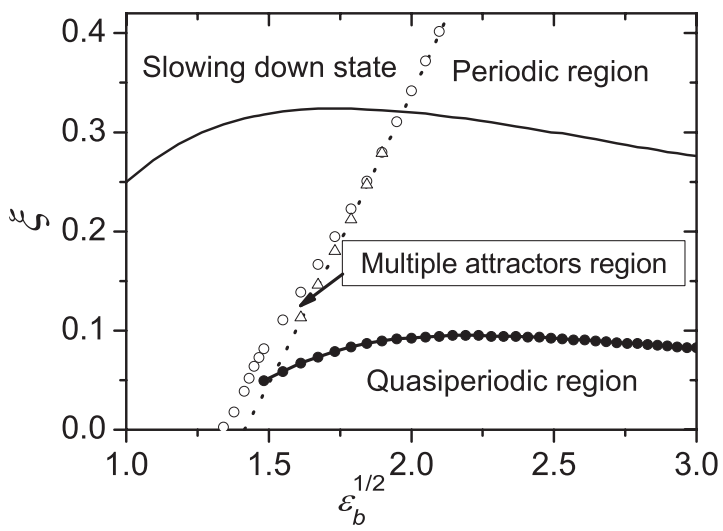

FIG. 7. Phase diagram varying $\xi$ and $\varepsilon_{b}$ while fixing $\tau_{a}=\tau_{b}=1$, $\varepsilon_{a}=1, g_{-}=0.5, g_{+}=2$. Filled circles are the Hopf bifurcation points from quasiperiodic to periodic oscillation, and unfilled circles are the critical points to the slowing-down state, both of which are numerically calculated with the initial conditions near the point $\left\{A_{1}=\right.$ $\left.A_{2}=A_{3}=\sqrt{\varepsilon_{a}} / 3, B_{1}=B_{2}=B_{3}=\sqrt{\varepsilon_{b}} / 3\right\}$. The triangles are the critical points to the slowing-down state when we use states near a saddle as the initial conditions for simulation. The dashed line is calculated from Eq. (14) $\left(\lambda_{21}+\lambda_{31}<0\right.$ to the left), and the solid line is $\lambda_{22}=0\left(\lambda_{22}<0\right.$ above the line $)$. Below the intersection of these two lines, the unfilled circles diverge from the dashed line. In the region between the unfilled circles and triangles multiple attractors coexist.

slowing-down state, as shown in Figs. 2 and 6. For smaller $\varepsilon_{b} \lesssim 3.880\left(\varepsilon_{b}^{1 / 2} \lesssim 1.970\right)$, on the other hand, $\lambda_{22}$ may still be positive when $\lambda_{21}+\lambda_{31}<0$ (the region below the solid line and above the dashed line in Fig. 7). For these values of $\varepsilon_{b}$, regions of multiple attractors may exist.

In the multiple-attractors regions, if we run a simulation using an initial condition near one of the saddles, we find the
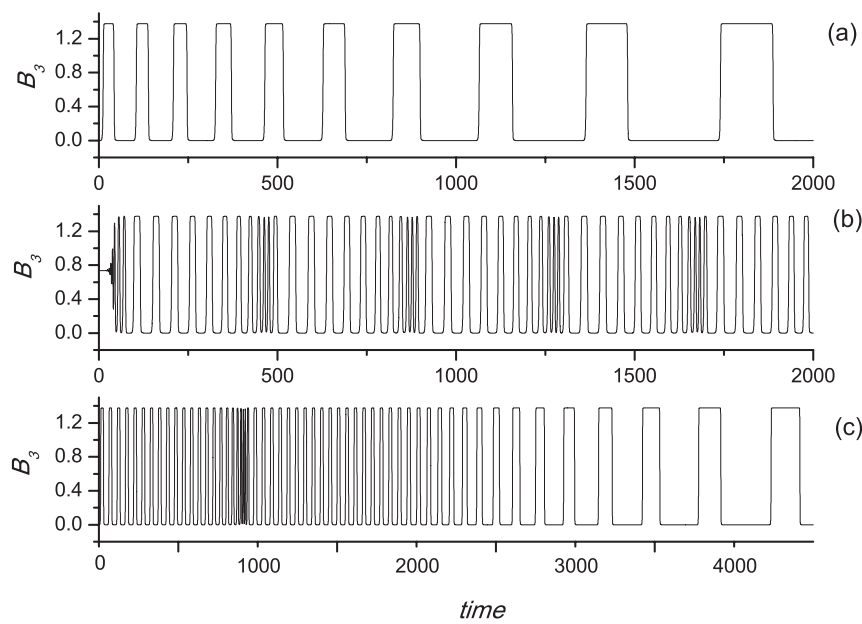

FIG. 8. An example of multiple attractors when $\tau_{a}=\tau_{b}=1$, $\varepsilon_{a}=1, \varepsilon_{b}=1.9, g_{-}=0.5, g_{+}=2, \xi=0.016$ : (a) slowing-down state with the initial condition near a saddle; (b) stable quasiperiodic orbit with the initial condition near $A_{1}=A_{2}=A_{3}=\sqrt{\varepsilon_{a}} / 3, B_{1}=$ $B_{2}=B_{3}=\sqrt{\varepsilon_{b}} / 3$. If the state shown in panel (b) is used as the initial condition and $\xi$ is increased to 0.01815 , the dynamics is driven to the attracting heteroclinic cycle, but the transient looks quite similar to quasiperiodic oscillation. This is shown in panel (c). 


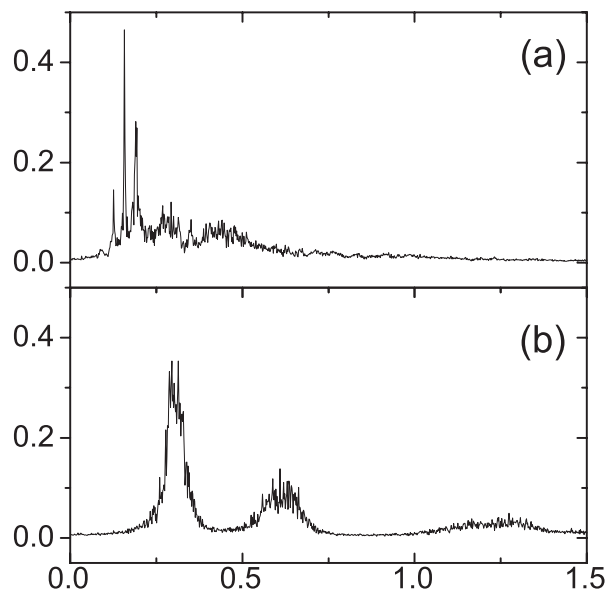

$\omega$

FIG. 9. Fourier transforms of one of the amplitudes in the noisy system for parameter values $\tau_{a}=\tau_{b}=1, \varepsilon_{a}=1, g_{-}=0.5, g_{+}=2$, and Gaussian noise intensity $10^{-10}$. Note the two base frequencies when $\varepsilon_{b}=1.9, \xi=0.02$ (a), but only a single base frequency when $\varepsilon_{b}=4, \xi=0.34$ (b).

critical points shown as the triangles in Fig. 7, which are well predicted by Eq. (14). But if we use an initial condition near the point $A_{1}=A_{2}=A_{3}=\sqrt{\varepsilon_{a}} / 3, B_{1}=B_{2}=B_{3}=\sqrt{\varepsilon_{b}} / 3$, we continue to find quasiperiodic or periodic attractors above the dashed line in Fig. 7 where $\lambda_{21}+\lambda_{31}<0$. Figure 8(a) and 8 (b) provide an example of the different attractors for the same parameter values.

When we use an initial condition near the point $A_{1}=A_{2}=$ $A_{3}=\sqrt{\varepsilon_{a}} / 3, B_{1}=B_{2}=B_{3}=\sqrt{\varepsilon_{b}} / 3$ for the numerical calculation, we can even find some cases in which the dynamics bifurcates from quasiperiodic oscillations to the slowing-down state without a region of periodic oscillations. This case happens when the destabilization of the quasiperiodic orbit occurs before the Hopf bifurcation. The unstable quasiperiodic orbit induces a quasiperiodic transient near the critical coupling, so that the slowing-down state takes the form of a slowing quasiperiodic oscillation, as in Fig. 8(c).

If we now add Gaussian noise to the system, the slowingdown state is avoided as for a single cycle, but the resulting behavior is sensitive to the location in the phase diagram. The Fourier transform of the absolute value of one of the amplitudes plotted in Fig. 9 shows the different possibilities. (We use the absolute value since the noise can cause transitions into the negative sectors of the amplitude space.) When the slowingdown state is near the boundary to a quasiperiodic oscillation and the transient to the slowing-down state in the absence of noise is quasiperiodic, two base frequencies are observed in the Fourier spectrum of the noisy system (panel (a)). However, near the boundary to a periodic orbit where the noise-free transient is periodic, there is only one base frequency (panel (b)).

\section{CONCLUDING REMARKS}

In this paper the dynamics of two coupled systems each showing a heteroclinic cycle has been investigated. Our findings reveal that, similar to the role of noise or diffusion, the coupling terms can prevent the heteroclinic cycles from slowing down. A variety of bifurcation and dynamical behavior has been observed, including the transition from a quasiperiodic state with two frequencies deriving from the two cycles to a periodic state in which the cycles are synchronized, via a Hopf bifurcation. This process does not happen through the commensurability of the two frequencies but from the disappearance of the weaker frequency oscillation. The two cycles play different roles in this process, with the master cycle dominating the dynamics and destroying the slave cycle. The synchronized frequency can be either larger or smaller than the individual desynchronized frequencies. When the coupling strength becomes too large, the dynamics of the coupled system fall back to the slowing-down state, as observed in the uncoupled (single-cycle) case. A resonant heteroclinic bifurcation occurs in this case. We have also found a regime where multiple attractors can coexist. Another observation is that sometimes the quasiperiodic oscillation can bifurcate directly from the heteroclinic cycle.

In this work, we demonstrate the rich variety of dynamical behavior by using the Busse-Heikes model with linear coupling, which is a typical heteroclinic cycle system with one of the simplest coupling forms used in coupled oscillatory systems. We have also simulated some other models with different forms of coupling, e.g., a model of two coupled three-competitor systems, and find similar results in some regions of the system parameters. In all the models studied, the symmetry of simultaneously interchanging the coordinates of the two subsystems in turn is always kept, even in the presence of the coupling terms, but the uncoupled systems are taken to be dissimilar. These two characteristics referring to the symmetry are important for the observed dynamical behavior. This asymmetry between the uncoupled systems is the basis for the quasiperiodic oscillation where the principal frequencies of the two oscillators induced by the coupling are nonidentical. It is also the basis for the observation that in the synchronized (periodic) state one oscillator can dominate, whereas the interchange symmetry retained plays a vital role in making the new heteroclinic cycle structurally stable.

Our results can give a precise understanding of the upward or downward shift or the synchronized frequency observed for coupled oscillators in neuronal system [5] and also shed some light on a similar phenomenon in magnetic system [46,47], and the rich dynamical behavior we find suggests further experiments in these and related systems. The dynamical behavior of two coupled heteroclinic cycles is also progress toward better understanding systems of a large number of cycles.

\section{ACKNOWLEDGMENTS}

The authors acknowledge Grigory Osipov for useful discussions. This work is supported in part by the National Natural Science Foundation of China, the 973 Program, the Foundation of Doctoral Training, and the Fundamental Research Funds for the Central Universities. M.C.C. thanks Beijing Normal University for support and hospitality during the initial stages of this work. C.Z. is supported by Hong Kong Baptist University. 
[1] C. M. Postlethwaite and J. H. P. Dawes, Nonlinearity 18, 1477 (2005).

[2] M. I. Rabinovich, R. Huerta, and P. Varona, Phys. Rev. Lett. 96, 014101 (2006).

[3] M. I. Rabinovich, R. Huerta, P. Varona, and V. S. Afraimovich, PLoS Comput. Biol. 4, e1000072 (2008).

[4] M. A. Komarov, G. V. Osipov, J. A. K. Suykens, and M. I. Rabinovich, Chaos 19, 015107 (2009).

[5] A. Szücs, R. Huerta, M. Rabinovich, and A. Selverston, Neuron 61, 439 (2009).

[6] M. A. Komarov, G. V. Osipov, and J. A. K. Suykens, Europhys. Lett. 86, 60006 (2009).

[7] M. A. Komarov, G. V. Osipov, and J. A. K. Suykens, Europhys. Lett. 91, 20006 (2010).

[8] F. H. Busse and K. E. Heikes, Science 208, 173 (1980).

[9] A. Demircan, S. Scheel, and N. Seehafer, Eur. Phys. J. B 13, 765 (2000).

[10] Y. Tu and M. C. Cross, Phys. Rev. Lett. 69, 2515 (1992).

[11] M. A. Nowak and K. Sigmund, Nature (London) 418, 138 (2002).

[12] B. Kerr, M. A. Riley, M. W. Feldman, and B. J. M. Bohannan, Nature (London) 418, 171 (2002).

[13] C. Hauert, S. De Monte, J. Hofbauer, and K. Sigmund, Science 296, 1129 (2002).

[14] M. A. Nowak and K. Sigmund, Science 303, 793 (2004).

[15] G. Szabó, T. Antal, P. Szabó, and M. Droz, Phys. Rev. E 62, 1095 (2000).

[16] C. Hauert and G. Szabó, Am. J. Phys. 73, 405 (2005).

[17] M. Doebeli and C. Hauert, Ecol. Lett. 8, 748 (2005).

[18] G. Szabó, J. Vukov, and A. Szolnoki, Phys. Rev. E 72, 047107 (2005).

[19] D. Li, W. Li, G. Hu, and Z. Zheng, Physica A 388, 1243 (2009).

[20] R. M. May and W. J. Leonard, SIAM J. Appl. Math. 29, 243 (1975).

[21] E. Stone and P. Holmes, SIAM J. Appl. Math. 50, 726 (1990).

[22] E. Stone and D. Armbruster, Chaos 9, 499 (1999).

[23] J. R. Gog, I. Oprea, M. R. E. Proctor, and A. M. Rucklidge, Proc. R. Soc. London A 455, 4205 (1999).

[24] S. A. Campbell and P. Holmes, Nonlinearity 4, 697 (1991).
[25] M. Krupa and I. Melbourne, Ergod. Theory Dyn. Syst. 15, 121 (1995).

[26] M. Tachikawa, Prog. Theor. Phys. 109, 133 (2003).

[27] P. L. Buono, M. Golubitsky, and A. Palacios, Physica D 143, 74 (2000).

[28] A. Pikovsky, M. Rosenblum, and J. Kurths, Synchronization: A Universal Concept in Nonlinear Sciences (Cambridge University Press, Cambridge, 2001).

[29] L. M. Pecora, T. L. Carroll, G. A. Johnson, D. J. Mar, and J. F. Heagy, Chaos 7, 520 (1997).

[30] S. Boccaletti, J. Kurths, G. Osipov, D. Valladares, and C. Zhou, Phys. Rep. 366, 1 (2002).

[31] L. M. Pecora and T. L. Carroll, Phys. Rev. Lett. 64, 821 (1990).

[32] N. F. Rulkov, M. M. Sushchik, L. S. Tsimring, and H. D. I. Abarbanel, Phys. Rev. E 51, 980 (1995).

[33] M. G. Rosenblum, A. S. Pikovsky, and J. Kurths, Phys. Rev. Lett. 76, 1804 (1996).

[34] Z. Liu and Y. C. Lai, Phys. Rev. Lett. 86, 4737 (2001).

[35] J. Yang, G. Hu, and J. Xiao, Phys. Rev. Lett. 80, 496 (1998).

[36] M. Zhan, G. Hu, and J. Yang, Phys. Rev. E 62, 2963 (2000).

[37] G. W. Wei, M. Zhan, and C.-H. Lai, Phys. Rev. Lett. 89, 284103 (2002).

[38] H. S. Horn and R. H. MacArthur, Ecology 53, 749 (1972).

[39] S. A. Levin, Am. Nat. 108, 207 (1974).

[40] M. Mimura, S. I. Ei, and Q. Fang, J. Math. Biol. 29, 219 (1991).

[41] J. A. Acebrón, L. L. Bonilla, C. J. P. Vicente, F. Ritort, and R. Spigler, Rev. Mod. Phys. 77, 137 (2005).

[42] B. Feng, Acta Math. Appl. Sinica 14, 404 (1998).

[43] M. Krupa, J. Nonlinear Sci. 7, 129 (1997).

[44] I. Melbourne, J. Dyn. Differ. Eq. 1, 347 (1989).

[45] See also Special Issue: Robust Heteroclinic and Switching Dynamics in Dynamical Systems, edited by V. Afraimovich, P. Ashwin, and V. Kirk, volume 25, issue 3, pages 285-443, 2010.

[46] J. Grollier, V. Cros, and A. Fert, Phys. Rev. B 73, 060409(R) (2006).

[47] D. Li, Y. Zhou, C. Zhou, and B. Hu, Phys. Rev. B 82, 140407(R) (2010). 